\title{
Remarked Suppression of A $\beta 42$ Protomer-Protomer Dissociation Reaction Elucidated by Molecular Dynamics Simulation
}

\author{
Ikuo Kurisaki ${ }^{1}$ and Shigenori Tanaka ${ }^{1}$ \\ ${ }^{1}$ Kobe Daigaku - Rokkodai Chiku Campus
}

October 19, 2021

\begin{abstract}
Multimeric protein complexes are molecular apparatuses to regulate biological systems and often determine their fate. Among proteins forming such molecular assemblies, amyloid proteins have drawn attention over a half-century since amyloid fibril formation of these proteins is supposed to be a common pathogenic cause for neurodegenerative diseases. This process is triggered by the accumulation of fibril-like aggregates, while the microscopic mechanisms are mostly elusive due to technical limitation of experimental methodologies in individually observing each of diverse aggregate species in the aqueous solution. We then addressed this problem by employing atomistic molecular dynamics simulations for the paradigmatic amyloid protein, amyloid- $\beta$ (1-42) (A $\beta_{42}$ ). Seven different dimeric forms of oligomeric $\mathrm{A} \beta_{42}$ fibril-like aggregate in aqueous solution, ranging from tetramer to decamer, were considered. We found additive effects of the size of these fibril-like aggregates on their thermodynamic stability and have clarified kinetic suppression of protomer-protomer dissociation reactions at and beyond the point of pentamer dimer formation. This observation was obtained from the specific combination of the $A \beta{ }_{42}$ protomer structure and the physicochemical condition that we here examined, while it is worthwhile to recall that several amyloid fibrils take dimeric forms of their protomers. We could thus conclude that the stable formation of fibril-like protomer dimer should be involved in a turning point where rapid growth of amyloid fibrils is triggered.
\end{abstract}

\section{Hosted file}

MainText__IK__2021_10_08_for_Proteins.docx available at https://authorea.com/users/441854/ articles/542291-remarked-suppression-of-a\%CE\%B2-42-protomer-protomer-dissociationreaction-elucidated-by-molecular-dynamics-simulation 